\title{
The Application Research of UAV-based LiDAR System for Power Line Inspection
}

\author{
Wei Zhang ${ }^{1, a}$, Xinqiao $\mathrm{Wu}^{1, \mathrm{~b}}$, Guifeng Zhang ${ }^{1, \mathrm{c}}$, Lei Ke $\mathrm{Ke}^{1, \mathrm{~d}}$, Liming Chen ${ }^{2, \mathrm{e},{ }^{*} \text {, }}$ \\ Xiao Chen ${ }^{1, \mathrm{f}}$, Hemeng Yang ${ }^{2, \mathrm{~g}}$, Xiaoming Qiao, ${ }^{2, \mathrm{~h}}$ and Yi Zhou ${ }^{2, \mathrm{i}}$ \\ ${ }^{1}$ Electric Power Rsearch Institute, CSG, Guangzhou 510080, China \\ ${ }^{2}$ Tianjin Zhongwei Aerospace Data System Technology Co. Ltd, Tianjin 300301, China \\ azhangwei2@csg.cn, bwuxq@csg.cn, czhanggf@csg.cn, dkelei919@163.com, \\ echen_dawn@163.com, f38122060@qq.com, ghustyhm@163.com, h342628651@qq.com, \\ i928512087@qq.com
}

Keywords: LiDAR, UAV, Power line surveys.

\begin{abstract}
Helicopter-based LiDAR system due to high input cost and personal risk resulting in a certain restrictions on overhead power lines. With the developed application of UAV platform for power lines surveys and miniaturization of LiDAR device, the integration of UAV-based LiDAR system comes to maturity and compensate for the lack of Helicopter platform. Unmanned helicopter based LiDAR system acquire high accuracy 3D point cloud to reconstruct the model of power lines and the dangerous point detection etc. In this article, the UAV-based LiDAR system utilizes the Dragon-50 unmanned helicopter, made in Switzerland, and GL-70 equipment, made in America. This system was used to survey the Shandong Zouchuan power line with $500 \mathrm{kV}$, acquired LiDAR point cloud successfully and met the expectation. The unmanned airborne LiDAR system provides a reference for the promotion of power inspection applications.
\end{abstract}

\section{Introduction}

With the gradually mature of airborne technology, photoelectric pod based on the airborne is gradually widely used in the power line survey, and achieved favorable effect [1-2]. Unfortunately, the distance between the power lines and the surface features on power line corridor is still cannot be measured [3]. Lidar (LiDAR) as a new type of photoelectric measurement equipment, provides a feasible solution to solve the problem of measurement in the power industry, gratified the development of high-voltage lines and smart grid construction [4]. LiDAR can obtain the three-dimensional information of geospatial space, combined with the advantages of airborne platform, making the airborne LiDAR system widely used, such as flood disaster investigation [5-6], power line project construction [7] and so on. Airborne LiDAR system has advantages in space positioning and accuracy measurement, which leads to a good application in the power line operation and maintenance aerial [3]. Therefore Airborne LiDAR system could apply in the dangerous corridors detection in the power line corridor, the distance measurement between the power lines precisely, power line three-dimensional visual management, detection the terrain changes of power line corridor, analysis the power line capacity, deforestation assessment and management of the tree on the corridor.

Airborne LiDAR technology achieved a breakthrough usage in the helicopter firstly, and promoted the application in China's power grid as a relatively mature inspection technology [8]. However, there are some inferiorities of helicopter inspection, such as one-time investment is very much, high inspection costs, high security risk of airborne personnel, the pilot's flight technical and psychological tolerance ability must be very good, airspace control complex and so on. Helicopter based Inspection technology is still not fully meet the needs of different voltage levels, complex terrain conditions and varieties of special disaster conditions. With the UAV technology is gradually maturing, the advantages are gradually obviously. Relative to the helicopter, UAV has some advantages, such as low manufacturing operating costs, mild landing space restrictions, simple 
personnel training, flexible flight and other characteristics [9]. UAV platform application in the field of power industry is gradually in-depth, and there are many researches relevant on the inspection technology [10-11]. LiDAR is becoming more and more lightweight, exalted performance and shrinking the volume. There has been some LiDAR can be applied on the UAV platform. For example, RIEGL has launched measurement level laser radar for the UAV system, provide the possible of LiDAR used on the UAV. This paper focuses on the application of unmanned helicopter and LiDAR system in power industry.

\section{Unmanned Airborne LiDAR System}

Unmanned helicopter has the character of vertical takeoff and landing, large load capacity and long flight time, which could provide a flexible inspection program for the overhead transmission lines [12]. LiDAR utilizes the Global Navigation Satellite System (GNSS) and the Inertial Navigation Module (IMU) to obtain high-precision position information and attitude information. LiDAR determines the location information of the target based on the object's reflection of the laser beam. LiDAR can record up to four echoes, such as trees, transmission lines and the final surface of the reflection echo and generate a point cloud from the collection of echo data. Unmanned airborne LiDAR system can obtain point cloud up to 100 per square meter for the ground and 10 per square meter for the transmission line. These densities of point cloud leads to $5 \sim 10 \mathrm{~cm}$ accuracy of the ground level and $2 \sim 5 \mathrm{~cm}$ accuracy of the elevation level.

The combined advantage of UAV and LiDAR form the unmanned airborne LiDAR system. The system accesses high-precision three-dimensional corridor terrain, features and lines facilities and equipment space information through the acquisition of high-precision line corridor three-dimensional laser point cloud and high-resolution aviation digital images. This system accurately and quickly measures the line corridor features (especially housing, trees, crossing across) to the wire distance, wire spacing and so on to judge whether those meet the safe operation requirements. The system reconstructs the power line model and detects the dangerous point. At the same time, high-resolution aviation digital images provide a strong help for the inspection staff to interpret the transmission lines and channel security risks and abnormalities [3].

The unmanned airborne LiDAR system used the Dragon-50 helicopter made in Switzerland as the UAV platform, and the GL-70 LiDAR produced by RIEGL USA as payload, as shown in Figure 1. During the process of system integration, the design of the damping system [13] reduced the distribution error of the laser spot cloud.

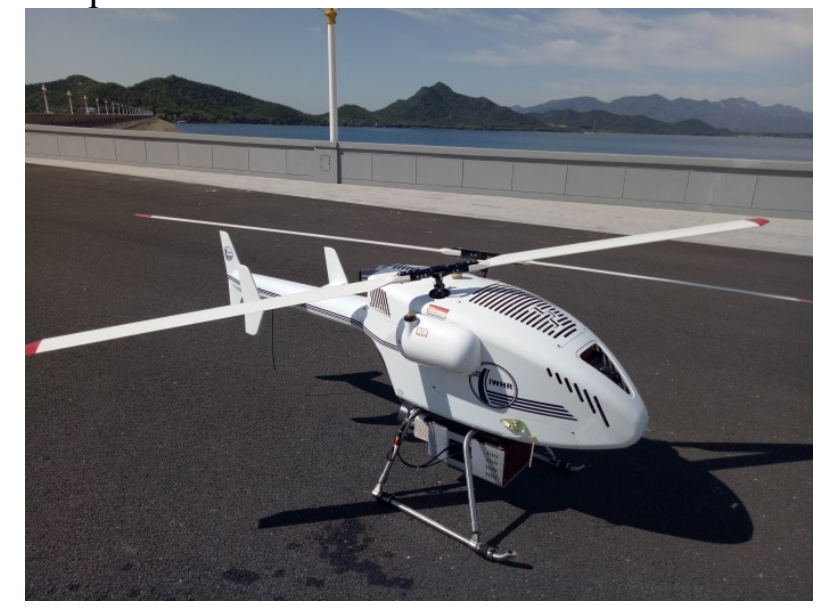

Fig. 1 Example of Dragon-50 mounting with GL-70 system integration.

\subsection{UAV platform}

Dragon-50 is a single-shot overlapped double-rotor unmanned aerial vehicle without tail rotor design to increase the load greatly and achieve the maximum load up to $50 \mathrm{~kg}$.

The platform of Dragon-50 is simple, stable and suitable for the implementation of hanging operations. The performance of Dragon-50 is excellent nearly without failure rate resulting in high 
service and simple on usage and maintenance. The product parameters list in Tab. 1. In addition, the integrated data link and the UAV platform leads to a long transmission distance, up to $50 \mathrm{~km}$.

Tab. 1 Parameters of Dragon-50

\subsection{LiDAR equipment}

\begin{tabular}{|c|c|}
\hline Parameter & Value \\
\hline Length & $2.17 \mathrm{~m}$ \\
\hline Width & $0.70 \mathrm{~m}$ \\
\hline Height & $0.98 \mathrm{~m}$ \\
\hline Rotor diameter & $2.8 \mathrm{~m}$ \\
\hline Empty load & $35 \mathrm{~kg}$ \\
\hline Maximum load & $85 \mathrm{~kg}$ \\
\hline Max. time of flight & $4 \mathrm{~h}$ \\
\hline Max. airspeed & $100 \mathrm{~km} / \mathrm{h}$ \\
\hline Max. height of flight(17kg payload) & $3 \mathrm{~km}$ \\
\hline Max. set number of points & 256 \\
\hline
\end{tabular}

The GL-70 airborne LiDAR consists of high-precision and mid-range laser scanners, high-precision airborne POS, high-resolution full-frame industrial cameras and control units. GL-70 synchronously accesses to laser and image data and supplies high-quality planning data products. Product technical parameters show in Table 2.

In order to monitor the working state of GL-70, installed the data link to download the high-definition video data in real time. In the ideal conditions, the data link's communication distance is about $30 \mathrm{~km}$ and the transmission rate is about $5 \mathrm{Mbps}$.

Tab. 2 Parameters of GL-70

\begin{tabular}{|c|c|}
\hline Parameter & Value \\
\hline Wavelength & Near infrared ray \\
\hline Laser beam divergence angle & $0.5 \mathrm{mrad}$ \\
\hline Range of distance & $5 \sim 900 \mathrm{~m}$ \\
\hline Max. scanning angle & $330^{\circ}$ \\
\hline Pulse frequency & $550 \mathrm{kHz}$ \\
\hline Max. of point cloud density & $400 \mathrm{pts} / \mathrm{m}^{2}$ \\
\hline Pitch angle accuracy & $0.015 \mathrm{deg}$ \\
\hline Roll angle accuracy & $0.015 \mathrm{deg}$ \\
\hline Heading accuracy & $0.03 \mathrm{deg}$ \\
\hline Imagine resolution & $8688 \times 5792$ \\
\hline Operating distance & $\begin{array}{c}\text { Powerline: } 100 \mathrm{~m} \\
\text { Others: } 350 \mathrm{~m}\end{array}$ \\
\hline Weight & $12 \mathrm{~kg}$ \\
\hline
\end{tabular}

\section{Application case}

The system was applied to inspect in Zouchuan line $500 \mathrm{kV}$ overhead transmission lines in December 2015 at Taian City, Shandong Province, with a total four flights.

\subsection{Flight design}

After determining the inspection line, select about $10 \times 10 \mathrm{~m}$ solid ground as a landing and takeoff site. Takeoff and landing point cannot have shrubs, trees and other vegetation within $100 \mathrm{~m}$ radius distance in order to ensure the safety of takeoff and landing; within the radius of $1 \mathrm{~km}$ surrounding, the field of vision is openness without obscure of mountains, hills or others to ensure the safe strength of the GPS signal. According to the account information, utilize the round station to design the track planning of the overhand transmission line.

As the inspection line section is located in the plains with flat terrain, we adopt the equal-height flight mode; In order to protect the line and system security, we utilize the obliquely above the power 
line flight mode. Considering the UAV hover accuracy and GPS horizontal coordinates error, the horizontal distance between the center of the transmission line and UAV platform is $30 \mathrm{~m}$ at least. In order to guarantee the point cloud density of the thin transmission line by the GL-70 collection quantity, the distance between the LiDAR and transmission should be within $100 \mathrm{~m}$. Take into account the average height of those inspected towers about $50 \mathrm{~m}$ high, therefore maximal safety height of the unmanned airborne LiDAR system from the average baseline is about $150 \mathrm{~m}$, which could meet the laser scanning measurements for the transmission.

In order to gather the maximum point cloud density of the ground reflection, the laser pulse frequency is set to the maximus pulse frequency of $550 \mathrm{kHz}$. At the same time, set the flight speed of UAV platform as $36 \mathrm{~km} / \mathrm{h}$. This takes into account the point cloud density and inspection efficiency. In this setting mode, the theoretical cloud density of GL-70 for the ground is about $58 \mathrm{pts} / \mathrm{m} 2$. The camera's exposure time interval is $7.9 \mathrm{~s}$, so there is enough overlapped part of image for post-processing. High-quality image data and high-density laser point cloud data leads to accurate modelling for the tower, wire and ground objects.

After adjust the UAV platform status, open the LiDAR data acquisition system and complete the parameters set, then according to the provisions of the flight path to collect data.

\subsection{Data processing and analysis}

After complete the data acquisition, the GPS data and laser point cloud data should be processed and preprocessed at the scene to confirm the reliability of the obtained data.

After the automatic classification of the point cloud $[14,15]$ and power lines extracted from the point cloud manually, the results could be got as shown in Fig. 2. The processed data shows that the horizontal accuracy is $10 \mathrm{~cm}$, the elevation accuracy is $5 \mathrm{~cm}$ and the point cloud density received from the ground reflection is more than $30 \mathrm{pts} / \mathrm{m}^{2}$.

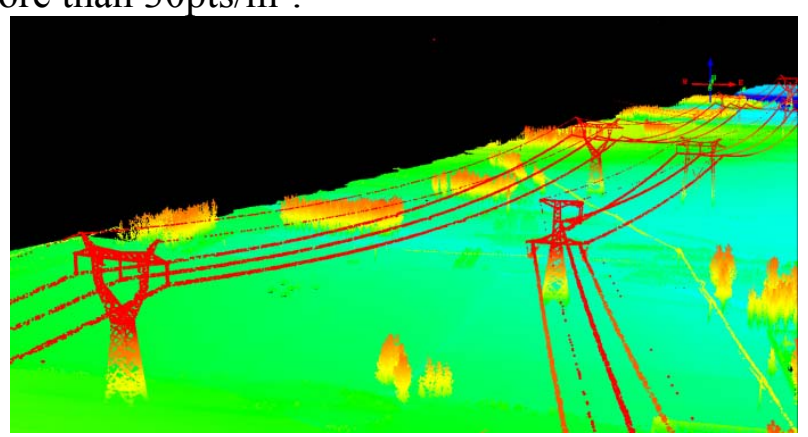

Fig. 2 Mapping of power line corridor with LiDAR point cloud data.

The processed data results show that the system obtains accurate three-dimensional information of the overhead transmission corridor, the distance between the tree barrier and power lines, deviation of power lines under the effect of wind and the crossing power lines information. The precision of these results is less than $10 \mathrm{~cm}$.

\section{Conclusion}

This paper validates the application of unmanned airborne LiDAR system in power line inspection, obtained high precision laser points cloud data successfully, carries out three-dimensional modeling and detected the dangerous points, achieves the expected effect. The low cost of UAV platform and the lightweight design of LiDAR provide a reference for the promotion of unmanned aerial carrier LiDAR in power industry application.

\section{References}

[1] SHEN Guang-sheng, ZHAO Xin-bo. Helicopter Power Line Inspection Tour Technology [J]. Electric Power Construction. 2008, 29(10):35-37.

[2] LIN Han, LIN Zhao-hui, TANG Ming-wen etc. Application of Unmanned Helicopter patrol to power Transmission line [J]. East China Electric Power, 2011, 39(10): 1657-1660. 
[3] YANG Feng, XU Zu-jian. Application of the Lidar Technology on Operation and Maintenance of Power Transmission Lines [J]. Southern Power System Technology, 2009, 3(2): 62-64.

[4] ZHANG Xian-feng, CHEN Gong, LONG Wei, et al. Current Status and Prospects of Helicopter Power Line Inspection Tour with LIDAR. Electric Power Construction, 2008, 29(3): 40-43.

[5] Zhang Qijian, Liu Changjun, Tang Xuezhe etc. Application of laser radar technique in flash flood disaster investigation in Luanchuan County. Mountain Flood Prevention, 2015, 25(5): 55-59.

[6] HU Xiaoqing, CHENG Penggen, NIE Yunju etc. Key Technology and Application of UAV Laser Radar in Torrential Disaster Investigation [J]. Jiangxi Science, 2016, 34(4): 470-474.

[7] SHU Long, LU Mang, HU Dan-hui. Application of LiDAR Technology in Power Line Selection [J]. Power System and Clean Energy, 2013, 29(1): 20-23.

[8] LI Guo-xing. Present Situation and Development of Helicopter Power Job in China. Electrical Equipment, 2006, 7(3): 41-45.

[9] LI Bingqiang, WANG Qian, WANG Binhai etc. Applying Unmanned Autonomous Helicopter to Transmission Line Inspection [J]. SHANDONG DIANLI JISHU, 2010(1), 172.

Geomatics and Information Science of Wuhan University

[10] TANGMing-wen, DAI Li-hao, LIN Chao-hui etc. Application of Unmanned Aerial Vehicle in Inspecting Transmission Lines [J]. 2013, 46(3): 35-38.

[11] PENG Xiangyang, QIAN Jinju, MAI Xiaoming etc. Automatic Power Line Inspection Technology of Large Unmanned Helicopter and Its Application [J]. Southern Power System Technology, 2016, 10(2): 24-31.

[12] Leena Matikainen, Matti Lehtomäki, Eero Ahokas. Remote sensing methods for power line corridor surveys [J]. Journal of Photogrammetry and Remote Sensing, 2016, 119: 10-31.

[13] LI Zhen-li, LIU Tao, LIU Tie etc. Shock Mitigation System Design and Error Analysis for UAV LiDAR System. Hubei Electric Power, 2016, 38(2): 51-54.

[14] YE Qingquan, WU Dezhi, LI Weihong. Powerline Danger Point Detection Using UAV LiDAR Point Cloud [J]. Mapping Report, 2015(11): 57-59.

[15] Bisheng Yang, Chi Chen. Automatic registration of UAV-borne sequent images and LiDAR data [J]. Journal of Photogrammetry and Remote Sensing, 2015, 101: 262-274. 